\title{
Centrality of trees for capacitated $k$-center
}

\author{
Hyung-Chan An · Aditya Bhaskara • Chandra Chekuri • \\ Shalmoli Gupta • Vivek Madan • Ola Svensson
}

Received: 30 April 2014 / Accepted: 17 December 2014 / Published online: 9 January 2015 (C) Springer-Verlag Berlin Heidelberg and Mathematical Optimization Society 2015

\begin{abstract}
We consider the capacitated $k$-center problem. In this problem we are given a finite set of locations in a metric space and each location has an associated nonnegative integer capacity. The goal is to choose (open) $k$ locations (called centers) and assign each location to an open center to minimize the maximum, over all locations, of the distance of the location to its assigned center. The number of locations
\end{abstract}

\begin{abstract}
The main result of this paper was obtained independently by An, Bhaskara and Svensson, and by Chekuri, Gupta and Madan. This paper is based on the manuscript of the first group. Work done while A. Bhaskara was at EPFL, Switzerland. C. Chekuri was supported in part by NSF Grants CCF-1016684 and CCF-1319376. H.-C. An and O. Svensson was supported in part by ERC Starting Grant 335288-OptApprox. A preliminary version of this work was presented in the 17th Conference on Integer Programming and Combinatorial Optimization.
\end{abstract}

H.-C. An $(\bowtie) \cdot$ O. Svensson

School of Computer and Communication Sciences,

École Polytechnique Fédérale de Lausanne, Lausanne, Switzerland

e-mail: hyung-chan.an@epfl.ch

O. Svensson

e-mail: ola.svensson@epfl.ch

\author{
A. Bhaskara \\ Google Research, New York, NY 10011, USA \\ e-mail: bhaskara@cs.princeton.edu \\ C. Chekuri · S. Gupta · V. Madan \\ University of Illinois at Urbana-Champaign, Champaign, IL 61801, USA \\ e-mail: chekuri@illinois.edu \\ S. Gupta \\ e-mail: sgupta49@illinois.edu \\ V. Madan \\ e-mail: vmadan2@illinois.edu
}


assigned to a center cannot exceed the center's capacity. The uncapacitated $k$-center problem has a simple tight 2-approximation from the 80 's. In contrast, the first constant factor approximation for the capacitated problem was obtained only recently by Cygan, Hajiaghayi and Khuller who gave an intricate LP-rounding algorithm that achieves an approximation guarantee in the hundreds. In this paper we give a simple algorithm with a clean analysis and prove an approximation guarantee of 9 . It uses the standard LP relaxation and comes close to settling the integrality gap (after necessary preprocessing), which is narrowed down to either 7,8 or 9 . The algorithm proceeds by first reducing to special tree instances, and then uses our best-possible algorithm to solve such instances. Our concept of tree instances is versatile and applies to natural variants of the capacitated $k$-center problem for which we also obtain improved algorithms. Finally, we give evidence to show that more powerful preprocessing could lead to better algorithms, by giving an approximation algorithm that beats the integrality gap for instances where all non-zero capacities are the same.

Keywords Approximation algorithms - Capacitated network location problems . Capacitated $k$-center problem $\cdot$ LP-rounding algorithms

\section{Mathematics Subject Classification 68W25}

\section{Introduction}

Network location problems form a large and important class of problems in discrete and combinatorial optimization. Many of these problems can be phrased in terms of choosing centers or facilities to best serve a given set of clients, typically under the assumption that the locations for the facilities and clients lie in a metric space. One can imagine several objective functions to measure the quality of service. Perhaps the most natural and well-studied ones are "social welfare", where we wish to minimize the average distance from a client to its assigned center, and "fairness", in which we wish to minimize the maximum distance from a client to its assigned center. Note that, once we have selected the centers, both of these objectives are minimized by assigning each client to its closest center. An inherent drawback of this strategy, however, is that it is unable to deal with centers of (different) capacities that limit the number of clients they can serve, which is a constraint present in typical applications. Capacity constraints in location problems pose difficult algorithmic challenges from both a theoretical and empirical point of view and our understanding continues to evolve despite a long history of work.

For uncapacitated network location problems, several beautiful algorithmic techniques, such as LP-rounding [7], primal-dual framework [16] and local search [6,18] have been used to obtain a fine-grained understanding of the approximability of the well-known variants: $k$-center, $k$-median, and facility location. ${ }^{1}$ Already in the 80 's,

\footnotetext{
1 Recall that in $k$-center and $k$-median, we wish to select $k$ centers so as to minimize the fairness and social welfare, respectively; facility location is similar to $k$-median but instead of having a constraint $k$ on the number of centers to open, each center has an opening cost.
} 
Gonzales [11], Hochbaum and Shmoys [14] developed tight 2-approximation algorithms for the $k$-center problem. For facility location, the current best approximation algorithm is due to Li [20]. He combined an algorithm by Byrka [5] and an algorithm by Jain et al. [15] to achieve an approximation guarantee of 1.488. This is nearly tight, as it is hard to approximate the problem within a factor of 1.463 [12]. The gap is slightly larger for $k$-median: a recent LP rounding [21] achieves an approximation guarantee of $1+\sqrt{3} \approx 2.732$ improving upon a local search algorithm by Arya et al. [2]; and it is NP-hard to do better than $1+2 / e \approx 1.736$ [15]. Although the different problems have algorithms with different approximation guarantees, they share many techniques, and improvements have often come hand in hand. In particular, most of the above progress relies on standard linear programming (LP) relaxations.

In contrast to the uncapacitated versions, the standard LP relaxations for the capacitated problems have unbounded integrality gaps and this is one reason for the coarser understanding we have. Apart from special cases, such as uniform capacities [17], soft capacities (a center can be opened several times) [16,17,23], and other variants $[9,19]$, the only known constant factor approximation algorithm until recently was for facility location. In a sequence of works, including Korupolu et al. [18], Pál et al. [22], Chudak and Williamson [8], and Zhang et al. [25], increasingly enhanced local search algorithms culminated in an approximation guarantee of 5 due to Bansal et al. [3]. These methods are elegant but specialized to facility location and are not LP-based. In fact, finding a relaxation-based algorithm for capacitated facility location with a constant approximation guarantee remains a major open problem (see e.g. "Problem 5" of the ten open problems from the recent book by Williamson and Shmoys [24]). One of the motivations for finding algorithms based on relaxations is that the methods are often flexible and the developed techniques transfer to different settings, as has indeed been the case in the study of uncapacitated location problems.

In the quest to obtain a better understanding and more general (relaxation based) techniques for capacitated network location problems, it is natural to start with the capacitated $k$-center problem. Indeed, even though we have a good understanding of uncapacitated location problems in general, the uncapacitated $k$-center problem stands out, with an extremely simple greedy algorithm that gives a tight analysis of the LP relaxation. Our failure to understand the capacitated $k$-center problem is therefore solely due to the lack of techniques for analyzing capacity constraints. An important recent development in this line of research is due to Cygan et al. [10], who obtain the first constant factor approximation for the capacitated $k$-center problem. Their algorithm works by preprocessing the instance to overcome the unbounded integrality gap of the natural LP relaxation, followed by an intricate rounding procedure. The approximation factor is not computed explicitly, but is estimated to be roughly in the hundreds. This however, is still quite far off from the integrality gap lower bound of 7 (after preprocessing) [10] and the inapproximability results which rule out a factor better than 3 (see e.g. [10] for a simple proof).

In this paper, we develop novel techniques to further close the gap in our understanding of capacitated location problems. In particular, we present a simple algorithm for the capacitated $k$-center problem with a clean analysis that allows us to prove an approximation guarantee of 9. Our result is based on the standard LP relaxation and it almost settles its integrality gap (after the preprocessing of Cygan et al. [10]): it is either 
7,8 or 9 (both the integrality gap and approximation ratio can only take integral values; this is because the worst instances can easily be seen to be ones defined by the shortestpath metric on an unweighted graph). Due to the simplicity of our analyses, we hope that some of the ideas could be applied to other location problems, such as capacitated $k$-median, for which no constant factor approximation algorithms are known.

Main result and outline of algorithmic approach. Our main result is the following.

Theorem 1 There exists a 9-approximation algorithm for the capacitated $k$-center problem.

The algorithm guesses the optimal solution value $\tau$ and considers an unweighted graph $G_{\leq \tau}$ on the given set of vertices where two vertices are adjacent if and only if their distance is at most $\tau$ : the edges in this graph represent the assignments that are "admissible" with respect to $\tau$. This graph can be assumed to be connected (see [10]). The algorithm then solves a natural and standard LP on $G_{\leq \tau}$. This determines if it is possible to (fractionally) open $k$ vertices while assigning every vertex to a center that is adjacent in $G_{\leq \tau}$. If this LP is infeasible, we know that the optimum is larger than $\tau$; otherwise, our algorithm will open $k$ centers and find an assignment of every vertex to an open center that is within a distance of 9 in $G_{\leq \tau}$, and moreover the assignment respects the capacities of open centers. This leads to a 9-approximation algorithm.

The LP solution specifies a set of opening variables that indicate the fraction to which each vertex is to be opened. Our algorithm rounds these opening variables by "transferring" openings between vertices to make them integral. Since we do not create any new opening, our rounding will naturally open at most $k$ centers; however, the challenge is to ensure that there exists a small-distance assignment of the vertices to open centers. If, for example, the opening of a vertex $v$ is transferred to another vertex that is far away, the clients that were originally assigned to $v$ may be unable to find an available center nearby. For another example, if the opening of a high-capacity vertex gets transferred to a low-capacity one, the low-capacity vertex may fail to provide sufficient capacity to cover the vertices in the neighborhood. Thus, we need to ensure that our rounding algorithm transfers openings only in small vicinity, and that "locally available capacity" of the graph does not decrease (Definition 1 formalizes this concept as a distance-r transfer.).

We reduce the rounding problem to the special case of tree instances, and present a best-possible algorithm that rounds such instances. A tree instance is given by a set of opening variables defined on a rooted tree, where every non-leaf node has an opening variable of 1 . Tree instances are generalizations of caterpillars used by Cygan et al. [10], which can be considered as tree instances whose non-leaf nodes form a path and have certain degree bounds. Suppose we have a tree instance where the capacities are uniform and there are exactly two leaves $u$ and $v$ each of which is opened to an extent of $1 / 2$, whereas every other vertex is opened by 1 . If $u$ and $v$ are distant, this may appear problematic at a glance as we cannot transfer the opening of one to the other. However, there exists a (unique) path $u, w_{1}, \ldots, w_{m}, v$ in the tree, and we can transfer the opening of $1 / 2$ in a "chain" along this path: from $u$ to $w_{1}$, from $w_{1}$ to $w_{2}$, ..., from $w_{m}$ to $v$. This idea can in fact be carried through to give an algorithm for capacitated $k$-center when all capacities are equal. 
Unfortunately, this chain of transfers can cause a problem under arbitrary capacities: suppose in the previous example that $u$ and $v$ have very high capacities compared to the others. Then we will not be able to transfer the opening of $u$ to $w_{1}$, since the open centers near $u$ may not be able to provide sufficient capacity to cover the vertices that were originally assigned to $u$. However, from a different viewpoint, $w_{1}$ (or any other non-leaf vertex) can be seen as "wasting" the budget, since it opens a center while contributing relatively small capacity to the graph. This provides us some "slack" in the budget that we can utilize: in this particular example, by transferring an opening of $1 / 2$ from $w_{1}$ to $u$, and the other $1 / 2$ from $w_{1}$ to $v$ in a chain, we can successfully round the given instance thanks to the decision of closing $w_{1}$ which had originally had its opening variable equal to one. This strategy of closing a fully open center is quite powerful, yet we need to ensure that its capacity can be accomodated by nearby centers if we want to close it. Thus, the viability of such a strategy tends to depend on several factors, including how its capacity compares to vertices in the neighborhood, which of these vertices are to be opened, and so on-all decisions which could depend on more and more distant vertices.

In contrast, our algorithm departs from previous works by using a simple local strategy that does not depend on distant vertices and applies to every non-leaf node. The reason our strategy works locally is that the decision of closing fully open centers is determined using solutions to subinstances, which are solved recursively. This key idea significantly eases the analysis and leads to our algorithm for tree instances that is the best possible. The simplicity of our analysis also helps us more carefully analyze the approximation ratio and extend our techniques to related problems. Section 4 formally presents our algorithm to round a tree instance; Sects. 6.1, 6.2 present its extensions to two related problems: the capacitated $k$-supplier problem and the budgeted opening problem with uniform capacity.

Finally, Sect. 3 presents our reduction to tree instances. We reduce the given problem to a tree instance by constructing a tree on a subset of vertices that are chosen as "candidates" to be opened. Non-leaf nodes will be carefully chosen, in order to yield a 9-approximation algorithm. Two adjacent vertices in the constructed tree instance will not necessarily be adjacent in the original graph, but will be in close proximity; hence, if the tree instance can be rounded using short transfers of openings, the original instance can also be rounded using only slightly longer transfers.

Additional results and future directions. We also explore future directions towards a better understanding of the problem. Recall that our algorithm proceeds in three steps: firstly, we preprocess the given instance as done by Cygan et al. [10]; secondly, we reduce the problem to a tree instance; lastly, we solve this tree instance. Given that our tree rounding algorithm is best-possible, it is natural to seek to improve the first two steps. The preprocessing step of Cygan et al. allows us to bring down the integrality gap from unbounded to 9; however, the integrality gap after the basic preprocessing is known to be at least 7 [10], which is larger than the best known inapproximability result that rules out a better factor than 3 . The instance showing the integrality gap of 7 (and also that of the inapproximability result) has a special structure that every capacity is either 0 or $L$ for some constant $L$. In order to understand the potential of stronger preprocessing methods, we investigate this $\{0, L\}$-case and show 
that additional preprocessing and a sophisticated rounding gives a 6-approximation algorithm (see Sect. 5). The interesting fact is that we obtain an approximation ratio which surpasses the integrality gap lower bound of 7 after basic preprocessing. This raises the natural open question: could there be preprocessing steps which bring the approximation ratio down to 3 ? We could also ask: do lift-and-project methods (applied to a potentially different formulation) automatically capture these preprocessing steps? We believe that understanding these questions would also shed light on approximating capacitated versions of other problems such as facility location and $k$-median.

\section{Preliminaries}

Given an integer $k$ and a metric distance/cost $c: V \times V \rightarrow \mathbb{R}_{+}$on $V$ with a capacity function $L: V \rightarrow \mathbb{Z}_{\geq 0}$, the capacitated $k$-center problem is to choose $k$ vertices to open, along with an assignment of every vertex to an open center which minimizes the longest distance between a vertex and the center it is assigned to while honoring the capacity constraints: i.e., no open center $v$ is assigned more vertices than its capacity $L(v)$.

For an undirected graph $G=(V, E), d_{G}(u, v)$ denotes the distance between $u, v \in$ $V ; N_{G}[u]$ denotes the set of vertices in the closed neighborhood of $u$, which includes $u$ itself: i.e., $N_{G}[u]:=\{v \mid(u, v) \in E\} \cup\{u\}$. For $U \subseteq V, d_{G}(v, U)$ denotes the distance from $v$ to $U: d_{G}(v, U):=\min _{u \in U} d_{G}(v, u)$. $N_{G}[U]$ is a shorthand for $\cup_{u \in U} N_{G}[u]$. When the graph of interest $G$ is clear from the context, we will use $d$ and $N[\cdot]$ instead of $d_{G}$ and $N_{G}[\cdot]$, respectively. Let OPT denote the optimal solution value.

Reduction to an unweighted problem using the standard LP relaxation. Our algorithm begins with determining a lower bound $\tau^{*}$ on the optimal solution value: it makes a guess $\tau$ at OPT, and tries to decide if $\tau<$ OPT. We simplify this problem by considering an unweighted graph that represents which assignments are "admissible". Let $G_{\leq \tau}=\left(V, E_{\leq \tau}\right)$ be the unweighted graph on $V$ (with loops on every vertex) where two vertices are adjacent if and only if their distance is at most $\tau: E_{\leq \tau}:=$ $\{(u, v) \mid c(u, v) \leq \tau\}$. Note that a feasible solution of value $\tau$ assigns every vertex to a center that is adjacent in $G_{\leq \tau}$, and conversely, if a solution assigns every vertex to a center that is adjacent in $G_{\leq \tau}$, its value is no greater than $\tau$. For an unweighted graph $G=(V, E)$, the standard LP relaxation $\operatorname{LP}_{k}(G)$ is the following feasibility LP that fractionally verifies whether there exists a solution that assigns every vertex to an open center that is adjacent in $G$ :

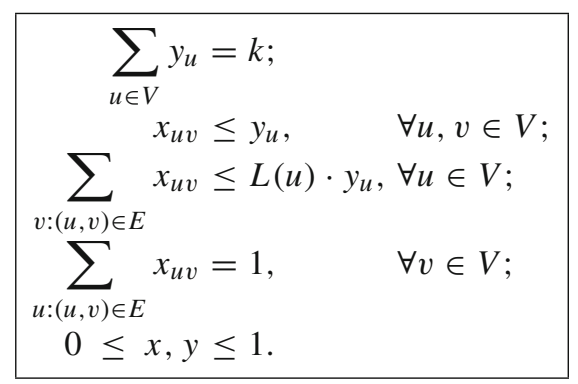

$x_{u v}$ is called an assignment variable; $y_{u}$ is called the opening variable of $u$. 
However, the integrality gap of this LP, defined as the maximum ratio $\frac{\mathrm{OPT}}{\tau}$ where $\operatorname{LP}_{k}\left(G_{\leq \tau}\right)$ is feasible, is unbounded; hence this LP cannot in general estimate OPT very well. We use the approach of Cygan et al. [10] to address this issue: consider the connected components of $G_{\leq \tau}$; if $\tau \geq \mathrm{OPT}$, a vertex can be assigned only to the vertices in the same connected component. For each connected component $G_{i}$ of $G_{\leq \tau}$, the algorithm decides the minimum integer $k_{i}$ for which $\operatorname{LP}_{k_{i}}\left(G_{i}\right)$ is feasible; if $\sum_{i} k_{i}>k$, this certifies that there exists no solution of value $\tau$ or better ( $\tau<$ OPT). Now let $\tau^{*}$ be the smallest $\tau$ for which the algorithm fails to certify that $\tau<$ OPT; since the algorithm has to fail to provide a certificate for $\tau=$ OPT, we have $\tau^{*} \leq$ OPT. The algorithm then separately solves the subproblems given by the connected components of $G_{\leq \tau^{*}}$ : given a connected graph $G$ for which $\operatorname{LP}_{k}(G)$ is feasible, our algorithm finds a set of $k$ vertices to open, with an assignment of every vertex to an open center that is within the distance of nine. Note that $d_{G_{\leq \tau^{*}}}(u, v) \leq 9$ implies $c(u, v) \leq 9 \tau^{*} \leq 9$. OPT from the triangle inequality.

Lemma 1 (Cygan et al. [10]) Suppose there exists an algorithm that, given a connected graph $G$, capacity $L$, and $k$ for which $\operatorname{LP}_{k}(G)$ is feasible, computes a set of $k$ vertices to open and an assignment of every vertex $u$ to an open center $v$ such that $d(u, v) \leq \rho$ and the capacity constraints are satisfied. Then we can obtain a $\rho$-approximation algorithm for the capacitated $k$-center problem.

Distance-r transfers. The above discussion reduces the task of designing an approximation algorithm for the capacitated $k$-center problem to that of using a solution $(x, y)$ to $\operatorname{LP}_{k}(G)$ in order to select $k$ centers so that each vertex in the connected graph $G$ is assigned to a center within a small distance. Simple algebraic manipulations show that, for any $U \subseteq V$, the LP solution satisfies $|U|=\sum_{u \in U} \sum_{w:(w, u) \in E} x_{w u} \leq$ $\sum_{w \in N[U]} L(w) \cdot y_{w}$; note that, if the opening variables $y$ are integral, this exactly corresponds to Hall's condition [13] and hence we can assign every vertex to an adjacent center. However, the LP solution may open each center only by a small fractional amount; in order to obtain an integral solution, it is therefore natural to try to aggregate fractional openings of nearby vertices. As different centers have varying capacities, one difficulty of this approach is that the rounding also needs to ensure that the aggregation does not decrease the available capacity. Consider a center $u$ of capacity $L(u)$ that is open with fraction $y_{u}$; we can view it as a center with the fractional capacity of $L(u) \cdot y_{u}$, because in a sense this is the maximum number (as a fraction) of vertices this center serves according to the LP. Our rounding procedure will open $k$ centers, while ensuring that we can transfer the fractional capacity of each $u$ to one or more of the open centers that are close by (and the performance guarantee is determined by how close these centers are). The following definition formalizes the notion of a distance- $r$ transfer:

Definition 1 Given a graph $G=(V, E)$ with a capacity function $L: V \rightarrow \mathbb{Z}_{\geq 0}$ and $y \in \mathbb{R}_{+}^{V}$, a vector $y^{\prime} \in \mathbb{R}_{+}^{V}$ is a distance-r transfer of $(G, L, y)$ if

(1a): $\sum_{v \in V} y_{v}^{\prime}=\sum_{v \in V} y_{v}$ and

(1b): $\sum_{v: d(v, U) \leq r} L(v) y_{v}^{\prime} \geq \sum_{u \in U} L(u) y_{u}$ for all $U \subseteq V$.

If $y^{\prime}$ is the characteristic vector of $S \subseteq V$, we say $S$ is a distance-r transfer of $(G, L, y)$. 
The given conditions say that a transfer should not change the total number of open centers, while ensuring that the total fractional capacity in each small neighborhood does not decrease as a result of this transfer. We also remark that multiple transfers can be composed: if $y^{\prime}$ is a distance- $r$ transfer of $(G, L, y)$ and $y^{\prime \prime}$ is a distance- $r^{\prime}$ transfer of $\left(G, L, y^{\prime}\right)$ then $y^{\prime \prime}$ is a distance- $\left(r+r^{\prime}\right)$ transfer of $(G, L, y)$.

Lemma 2 For a graph $G=(V, E)$ with a capacity function $L: V \rightarrow \mathbb{Z}_{\geq 0}$, let $(x, y)$ be a feasible solution to $\operatorname{LP}_{k}(G)$. If $S \subseteq V$ is a distance-r transfer of $(G, L, y)$, then every vertex $v \in V$ can be assigned to a center $s \in S$ such that $d_{G}(v, s) \leq r+1$, while ensuring no center is assigned more vertices than its capacity. Moreover, $|S|=k$, and this assignment can be found in polynomial time.

Proof Consider the natural bipartite matching problem between $V$ and the multiset of open centers that are duplicated to their capacities: i.e, each center $s \in S$ appears in the multiset with multiplicity $L(s)$. Every vertex $v$ in $V$ is connected to every copy of each center $s \in S$ such that $d(v, s) \leq r+1$. Observe that a matching of cardinality $|V|$ naturally defines an assignment that satisfies the desired properties. We shall now show that there exists such a matching by verifying Hall's condition, i.e., that for all $U \subseteq V,|U| \leq \sum_{s \in S: d_{G}(s, U) \leq r+1} L(s)$.

As was observed earlier, we have $|U| \leq \sum_{w: d_{G}(w, U) \leq 1} L(w) \cdot y_{w}$; from Condition (1b), $|U| \leq \sum_{w: d_{G}(w, U) \leq 1} L(w) \cdot y_{w} \leq \sum_{s \in S: d_{G}(s, U) \leq r+1} L(s)$. This matching can be found in polynomial time, and $|S|=k$ follows from Condition (1a).

Tree instances. As was discussed earlier, we solve the general problem via reduction to tree instances.

Definition 2 A tree instance is defined as a tuple $(T, L, y)$, where $T=(V, E)$ is a rooted tree with the capacity function $L: V \rightarrow \mathbb{Z}_{\geq 0}$, and opening variables $y \in$ $(0,1]^{V}$ satisfy that $\sum_{v \in V} y_{v}$ is an integer and $y_{v}=1$ for every non-leaf node $v \in V$.

\section{Reducing general instances to trees}

In this section, we present the reduction from the capacitated $k$-center problem to tree instances.

Lemma 3 Suppose there exists a polynomial-time algorithm that finds an integral distance-r transfer of a tree instance. Then there exists a $(3 r+3)$-approximation algorithm for the capacitated $k$-center problem.

Lemma 3 directly follows from Lemmas 1, 2, and 4.

Lemma 4 Suppose there exists a polynomial-time algorithm that finds an integral distance-r transfer of a tree instance. Then there exists an algorithm that, given a connected graph $G=(V, E)$, capacity $L: V \rightarrow \mathbb{Z}_{\geq 0}$, and $k \in \mathbb{N}$ for which $\operatorname{LP}_{k}(G)$ has a feasible solution $(x, y)$, finds an integral distance- $(3 r+2)$ transfer of $(G, L, y)$.

Our reduction, conceptually, constructs a tree instance by defining a tree on a subset of the vertices that have nonzero opening variables in the LP solution. Adjacent vertices 
in this tree instance may not necessarily be adjacent in $G$, but will be in close proximity; this establishes that a distance- $r$ transfer of the tree instance can be interpreted as a short-distance transfer in $G$. The opening variables of this tree instance would ideally be set equal to the corresponding LP opening variables. However, recall that one of the crucial characteristics of tree instances is that every non-leaf node has the opening variable of one. Yet, individual opening variables of the LP solution may have values less than one in general; we address this issue by using the clustering due to Khuller and Sussmann [17].

Lemma 5 (Khuller and Sussmann [17]) Given a connected graph $G=(V, E), V$ can be partitioned into $\left\{C_{v}\right\}_{v \in \Gamma}$ for some set of cluster midpoints $\Gamma \subseteq V$, such that

- there exists a tree $U=(\Gamma, F)$ rooted at $r \in \Gamma$ such that for every $(u, v) \in F$, $d_{G}(u, v)=3$;

- for all $v \in \Gamma, N_{G}[v] \subseteq C_{v}$; and

- for all $u \in C_{v}, d_{G}(u, v) \leq 2$.

Proof (sketch, [17]) Take an arbitrary node as $r$. We iteratively choose additional cluster midpoints as follows: for each vertex $v$, compute the shortest distance from $v$ to any of the cluster midpoints chosen so far. If every vertex is within distance 2 from the current set of cluster midpoints, we are done choosing midpoints. Otherwise, there exists a vertex $v$ that is at distance 3 from a cluster midpoint $w$ (and no shorter from the other midpoints); choose $v$ as a new cluster midpoint, make it a child of $w$ in the tree, and repeat.

Note that every pair of chosen midpoints are at distance 3 or more; hence, their closed neighborhoods are disjoint. Moreover, every vertex is within a distance of 2 from some midpoint. This shows that there exists a valid partition $\left\{C_{v}\right\}_{v \in \Gamma}$.

Observe that, for every cluster $C_{v}$, the total opening in the neighborhood of $v$ is at least one: $\sum_{u \in N_{G}[v]} y_{u} \geq \sum_{u \in N_{G}[v]} x_{u v}=1$ from the LP constraints. We will aggregate these openings to create at least one vertex with the opening variable of one in each cluster; then each cluster will contribute one "fully open vertex" to the tree instance, which will become the non-leaf nodes of the tree. Two non-leaf nodes in the tree instance are made adjacent if and only if their clusters are adjacent in $U$. In order to ensure that the aggregation retains the fractional capacity in the graph [in other words, to satisfy Condition (1b) of Definition 1], we will transfer the openings in $N_{G}[v]$ to a vertex with the highest capacity in $N_{G}[v]$. Let $m_{v}:=\operatorname{argmax}_{u \in N_{G}[v]} L(u)$ denote this vertex.

If $m_{u}$ and $m_{v}$ are adjacent in this tree instance, how far can they be in $G$ ? Recall that $m_{u}$ and $m_{v}$ are adjacent if and only if $(u, v) \in F$; hence, $d_{G}\left(m_{u}, m_{v}\right) \leq d_{G}\left(m_{u}, u\right)+$ $d_{G}(u, v)+d_{G}\left(v, m_{v}\right) \leq 5$. However, here comes a subtlety: if $m_{v}$ and $m_{w}$ are also adjacent in the tree, we would expect $d_{G}\left(m_{u}, m_{w}\right) \leq d_{G}\left(m_{u}, m_{v}\right)+d_{G}\left(m_{v}, m_{w}\right) \leq$ 10, whereas a tighter bound shows that $d_{G}\left(m_{u}, m_{w}\right)$ in fact never exceeds 8: $d_{G}\left(m_{u}, m_{w}\right) \leq d_{G}\left(m_{u}, u\right)+d_{G}(u, v)+d_{G}(v, w)+d_{G}\left(w, m_{w}\right) \leq 1+3+3+1$. Therefore, a simple abstraction that a tree edge corresponds to a length-5 path in $G$ would lead to a slight slack in the analysis. In order to avoid this issue, we will create an auxiliary vertex $a_{v}$ that is "almost at the same position" as the cluster midpoint $v$ for each cluster, and aggregate openings to this auxiliary vertex $a_{v}$ instead of $m_{v}$ as we 
did earlier. We will treat $a_{v}$ as the delegate for $m_{v}$, in the sense that $a_{v}$ (in lieu of $m_{v}$ ) will be part of our tree instance, and if we decide to open $a_{v}$ from the tree instance, we will open $m_{v}$ instead.

Proof (of Lemma 4) We begin by invoking Lemma 5 to obtain a partition of vertices $\left\{C_{v}\right\}_{v \in \Gamma}$ and a tree $U=(\Gamma, F)$ of the cluster midpoints. Recall that, for each $v \in \Gamma$, $m_{v}:=\operatorname{argmax}_{u \in N_{G}[v]} L(u)$ denotes a vertex with the highest capacity in $N_{G}[v]$.

We first augment the graph by introducing the auxiliary vertices (see also Fig. 1): for each $C_{v}$, we add a new vertex $a_{v}$ to the graph, along with the edges from $a_{v}$ to every vertex in $N_{G}[v]$. Let $\bar{G}=(\bar{V}, \bar{E})$ be this augmented graph. Observe that $a_{v}$ is located "almost at the same position" as $v$ in the following sense: for every $u \in V$, $d_{\bar{G}}\left(u, a_{v}\right)=d_{G}(u, v)$ unless $u=v ; d_{\bar{G}}\left(v, a_{v}\right)=1$. Note that $d_{\bar{G}}\left(a_{w}, a_{z}\right)=d_{G}(w, z)$. $L$ and $y$ are accordingly augmented by setting the capacity and the opening variable of the new auxiliary vertex respectively as $L\left(a_{v}\right):=L\left(m_{v}\right)$ and $y_{a_{v}}:=0$.

Now our reduction works in three phases: in the first phase, we aggregate the opening of 1 from $N_{G}[v]$ to $a_{v}$; this phase yields a distance- 1 transfer $y^{\text {first }}$ of $(\bar{G}, L, y)$. In the second phase, we construct a tree instance by defining a tree on a subset of $\bar{V}$, and invoke the polynomial-time algorithm to find an integral distance- $r$ transfer of this tree instance. We will see that this transfer can be interpreted as a distance- $3 r$ transfer $y^{\text {second }}$ of $\left(\bar{G}, L, y^{\text {first }}\right)$. In the last phase, we transfer the opening of each auxiliary variable $a_{v}$ to the vertex it delegates, $m_{v}$. This constitutes a distance- 1 transfer $y^{\text {third }}$ of $\left(\bar{G}, L, y^{\text {second }}\right)$.

The opening aggregation in the first phase works as follows: for each cluster $C_{v}$, we increase $y_{a_{v}}$ while simultaneously decreasing $y_{u}$ for some $u \in N_{G}[v]$ with $y_{u}>0$. If $y_{a_{v}}$ reaches one, we stop; if $y_{u}$ reaches zero, we find another $u \in N_{G}[v]$. The initial choice of $u$ is always taken as $m_{v}$ so that this procedure ensures that $y_{m_{v}}$ becomes zero. The procedure outputs a distance- 1 transfer $y^{\text {first }}$, since whenever an opening variable decreases during the construction, we increase the opening variable of an adjacent vertex with higher or equal capacity.

In the second phase, we define a tree $T$ on the set of vertices with nonzero opening variables. Note that this in particular implies that $m_{v} \notin T$ for each cluster $C_{v} . T$ is constructed from $U=(\Gamma, F)$ as follows: we replace each $v \in \Gamma$ by $a_{v}$ to obtain a tree on the auxiliary vertices, and for each vertex $u \in C_{v}$ such that $y_{u}>0$, we attach $u$ as a (leaf) child of $a_{v}$. Note that every non-leaf node is an auxiliary vertex and therefore has the opening variable of one. The total opening is equal to the total opening of $y$, and therefore $\left(T, L, y^{\mathrm{first}}\right)$ is a valid tree instance; we invoke the polynomial-time
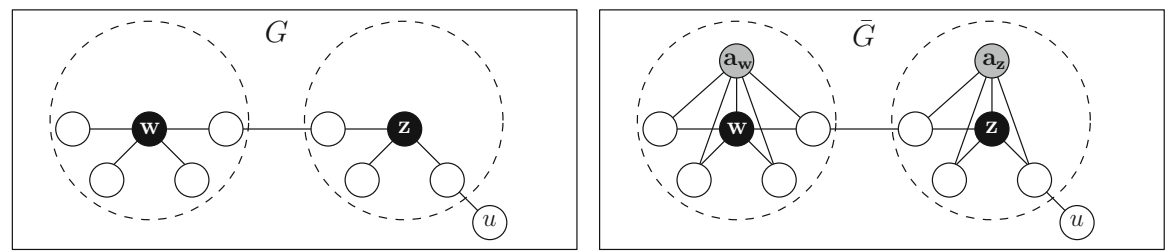

Fig. 1 Graph $\bar{G}$ obtained by augmenting $G$ with auxiliary vertices; black nodes correspond to cluster midpoints, dashed circles represent their neighborhoods 
algorithm to find an integral distance- $r$ transfer of this instance. For any two nodes $i$ and $j$ that are adjacent in this tree instance, either $i=a_{u}$ and $j=a_{v}$ for some $(u, v) \in F$, or $i=a_{v}$ and $j \in C_{v}$. In the former case, $d_{\bar{G}}(i, j)=3$; in the latter case, $d_{\bar{G}}(i, j) \leq 2$. Thus, the integral distance- $r$ transfer of the tree instance can be interpreted as an integral distance- $3 r$ transfer $y^{\text {second }}$ of $\left(\bar{G}, L, y^{\text {first }}\right)$.

Note that $y_{m_{v}}^{\text {second }}=0$ for every cluster $C_{v}$, since $m_{v}$ does not participate in the tree instance; on the other hand, $a_{v}$ may have been opened by the tree algorithm. In the last phase, we transfer the opening of $a_{v}$ to $m_{v}$, the vertex delegated by $a_{v}$. This yields an integral distance- 1 transfer $y^{\text {third }}$ of $\left(\bar{G}, L, y^{\text {second }}\right)$.

Note that $y_{a_{v}}^{\text {third }}=0$ for every cluster $C_{v}$; by projecting $y^{\text {third }}$ back to $V$, we obtain an integral distance- $(3 r+2)$ transfer of $(G, L, y)$.

\section{Algorithm for tree instances}

In this section we prove the following.

Lemma 6 There is a polynomial time algorithm that finds an integral distance-2 transfer of a given tree instance $(T, L, y)$.

We remark that it is easy to see that some tree-instances do not admit an integral distance- 1 transfer and the above lemma is therefore the best possible. One example is the following: the instance consists of a root with six children, where each child is opened with a fraction $2 / 3$, and all vertices have the same capacity; it is easy to see that any integral solution needs to transfer fractional capacity from one leaf to another (i.e., of distance 2). We now present the algorithm along with the arguments of its correctness.

The algorithm builds up the solution by recursively solving smaller tree instances. The base case is simple: if $|T| \leq 1$ then simply open the vertex in $V(T)$ if any. By the integrality of $\sum_{v \in V(T)} y_{v}$ this is clearly a distance-2 transfer (actually a distance-0 transfer). Let us now consider the more interesting case when $|T| \geq 2$; then there exists a node $r$ of which every child is a leaf. Let $v_{1}, \ldots, v_{\ell}$ be the children of $r$, in the non-increasing order of capacity: $L\left(v_{1}\right) \geq \cdots \geq L\left(v_{\ell}\right)$. Let $T_{r}$ denote the subtree rooted at $r$ and $Y:=\sum_{i=1}^{\ell} y_{v_{i}}$. The algorithm considers two separate cases depending on whether $Y$ is an integer.

Let us start with the simpler case when $Y$ is an integer: the algorithm selects the set $S_{r}$ consisting of the $Y+1$ vertices of highest capacity in $T_{r}$. Recall that $y_{r}=1$ and therefore $\sum_{v \in T_{r}} y_{v}=Y+1$; as every pair of nodes in $T_{r}$ are within a distance of $2, S_{r}$ is a distance- 2 transfer of the tree instance induced by $T_{r}$. The algorithm then solves the tree instance induced by $\bar{T}:=T \backslash T_{r}$ to obtain a distance- 2 transfer $\bar{S}$ of size $\sum_{v \in T} y_{v}-Y-1$. It follows that $S:=S_{r} \cup \bar{S}$ is a distance-2 transfer of $(T, L, y)$.

We now consider the final more interesting case when $Y$ is not an integer. In this case, we cannot consider $T_{r}$ and $T \backslash T_{r}$ as two separate instances because the $y$-values suggest to either open $\lfloor Y\rfloor+1$ or $\lceil Y\rceil+1$ centers in $T_{r}$ : a choice that depends on the selected centers in $T \backslash T_{r}$. As at least $\lfloor Y\rfloor+1$ of the vertices in $T_{r}$ will be selected as centers in either case, the algorithm will commit itself to open the $\lfloor Y\rfloor+1$ vertices in $T_{r}$ of highest capacity. Let $S_{\text {commit }}$ denote that set and it is chosen as either $\left\{v_{1}, \ldots, v_{\lfloor Y\rfloor}, r\right\}$ 
or $\left\{v_{1}, \ldots, v_{\lfloor Y\rfloor}, v_{\lfloor Y\rfloor+1}\right\}$ dependent on which node of $r$ and $v_{\lfloor Y\rfloor+1}$ has the higher capacity $\left(v_{\lfloor Y\rfloor+1}\right.$ is well defined since we have that the number of children $\ell$ is at least $\lceil Y\rceil$ from $y \leq \mathbf{1})$. By the selection of $S_{\text {commit }}$, we have

$$
\sum_{u \in V\left(T_{r}\right)} y_{u} L(u) \leq \sum_{s \in S_{\text {commit }}} L(s)+\bar{y}_{p} \bar{L}(p),
$$

where $\bar{y}_{p}=Y-\lfloor Y\rfloor$ and $\bar{L}(p)=\min \left[L(r), L\left(v_{\lfloor Y\rfloor+1}\right)\right]$. In other words, if the algorithm on the one hand chooses to only open the $\lfloor Y\rfloor+1$ centers $S_{\text {commit }}$ in $T_{r}$, then an additional fractional capacity $\bar{y}_{p} \bar{L}(p)$ needs to be transferred from $T_{r}$ to an open center in $T \backslash T_{r}$. On the other hand, if the algorithm chooses to open all the centers $\lceil Y\rceil+1$ in $S_{\text {commit }} \cup\left\{v_{\lfloor Y\rfloor+1}, r\right\}$ then those centers can accomodate all the fractional capacity in $T_{r}$ together with $\left(1-\bar{y}_{p}\right) \bar{L}(p)$ additional capacity.

We defer this decision to be based on the solution of the smaller tree instance ( $\bar{T}, \bar{L}, \bar{y}$ ) obtained from (T, $L, y$ ) as follows (see also Fig. 2a): replace $T_{r}$ by the vertex $p$ that represents the deferred decision and let $\bar{y}, \bar{L}$ be the natural restrictions of $y, L$ on $T \backslash T_{r}$ with $\bar{y}_{p}=Y-\lfloor Y\rfloor$ and $\bar{L}(p)=\min \left[L(r), L\left(v_{\lfloor Y\rfloor+1}\right)\right]$. The algorithm then recursively solves this smaller instance to obtain a distance-2 transfer $\bar{S}$ of $(\bar{T}, \bar{L}, \bar{y})$. From $\bar{S}$ it constructs the solution $S$ to the original problem instance by first replacing $p$ (if $p$ is in $\bar{S}$ ) by the vertex $v_{\lfloor Y\rfloor+1}$ or $r$ that was not chosen to be in $S_{\text {commit, }}$, and then adding $S_{\text {commit }}$ to the set.

We complete the proof of Lemma 6 by arguing that $S$ is a distance- 2 transfer of the original tree instance $(T, L, y)$. Note that, as $|\bar{S}|=\sum_{v \in \bar{T}} \bar{y}_{v}=\sum_{v \in T} y_{v}-1-\lfloor Y\rfloor$, we have $|S|=|\bar{S}|+\left|S_{\text {commit }}\right|=\sum_{v \in V} y_{v}$ as required. It remains to verify Condition (1b) of Definition 1:

Claim 2 We have $\sum_{u \in U} y_{u} L(u) \leq \sum_{s \in S: d_{T}(s, U) \leq 2} L(s)$ for all $U \subseteq V(T)$.

Proof Consider the bipartite graph $G$ with left-hand-side $V(T)$, right-hand-side $S$, and an edge between $v \in V(T)$ and $s \in S$ if $d_{T}(s, v) \leq 2$. For simplicity, we slightly abuse notation and think of $V(T)$ and $S$ as disjoint sets. Moreover, let $N(U)$ denote the (open) neighbors of a subset $U$ of vertices in this graph: $N(U):=\left\{v \mid \exists u \in U d_{G}(u, v)=1\right\}$.

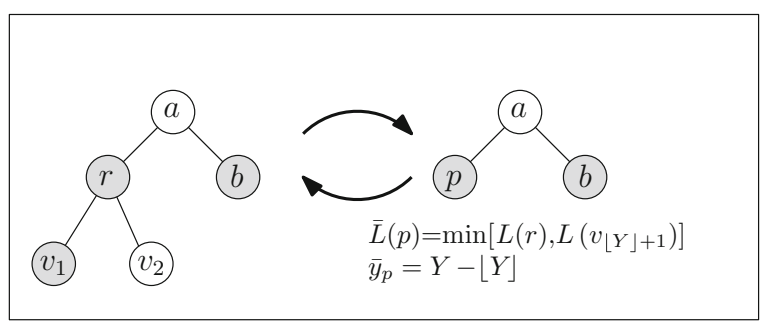

(a)

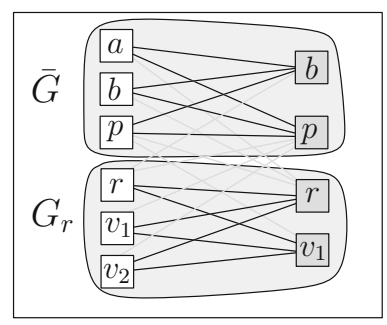

(b)

Fig. 2 a The construction of $\bar{T}$ from $T$ with the subtree $T_{r}$ rooted at $r$ with children $v_{1}$ and $v_{2}$; the grey vertices are those selected in potential solutions to $\bar{T}$ and $T$, respectively. b The bipartite graph and the induced subgraphs $\bar{G}$ and $G_{r}$ that are used in the proof of Claim 2 
Let $w: V(T) \cup S \rightarrow \mathbb{R}$ be weights on the vertices defined by

$$
w(v)=\left\{\begin{array}{ll}
y_{v} L(v) & \text { if } v \in V(T) \\
L(v) & \text { if } v \in S
\end{array} .\right.
$$

With this notation, we can reformulate the condition of the claim as

$$
\sum_{u \in U} w(u) \leq \sum_{s \in N(U)} w(s) \quad \text { for all } U \subseteq V(T) .
$$

To prove this, we shall prove a slightly stronger statement by verifying the condition separately on two biparite graphs $G_{r}$ and $\bar{G}$ that correspond to $T_{r}$ and $\bar{T}$, respectively. We obtain $G_{r}$ and $\bar{G}$ from $G$ as follows (see also Fig. 2b). First, add a vertex $p$ to the left-hand-side by making a copy of $r \in T$ and set $w(p)=\bar{y}_{p} \bar{L}(p)$ and update $w(r)=y_{r} L(r)-\bar{y}_{p} \bar{L}(p) \geq 0$, where the inequality follows from $y_{r}=1$ and $\bar{L}(p) \leq L(r)$. Similarly, if $p \in \bar{S}$ then add a copy $p$ of $r \in S$ and set $w(p)=\bar{L}(p)$ and update $w(r)=L(r)-\bar{L}(p) \geq 0$. Note that after these operations the vertices of both the left-hand-side and the right-hand-side can naturally be partitioned into those that correspond to vertices in $T_{r}$ and those that correspond to vertices in $\bar{T}$. Graphs $G_{r}$ and $\bar{G}$ are the subgraphs induced by these two partitions.

Let us first verify that (2) holds for $\bar{G}$. By construction, we have that the total weight $w(U)$ of a subset $U$ of $V(\bar{T})$ is equal to $\sum_{u \in U} \bar{y}_{u} \bar{L}(u)$ and the total weight $w(N(U))$ of its neighborhood in $\bar{G}$ equals $\sum_{s \in \bar{S}: d_{T}(s, U) \leq 2} \bar{L}(s)$. Hence, (2) holds since $\bar{S}$ is a distance-2 transfer of $(\bar{T}, \bar{L}, \bar{y})$.

We conclude the proof of the claim by verifying (2) for $G_{r}$. As both the left-handside and right-hand-side of $G_{r}$ correspond to vertices in $T_{r}$ that all are within distance 2 of each other, we have that $G_{r}$ is a complete bipartite graph. The total weight of the left-hand-side vertices is by construction $\sum_{u \in T_{r}} y_{u} L(u)-\bar{y}_{p} \bar{L}(p)$ and the total weight of the right-hand-side is $\sum_{s \in T_{r} \cap S} L(s)-\bar{L}(p) \mathbf{1}_{p \in \bar{S}}=\sum_{s \in S_{\text {commit }}} L(s)$, where $\mathbf{1}_{p \in \bar{S}}$ is defined as 1 if $p \in \bar{S}$ and 0 otherwise. The claim now follows from (1), i.e., that $\sum_{u \in T_{r}} y_{u} L(u)-\bar{y}_{p} \bar{L}(p) \leq \sum_{s \in S_{\text {commit }}} L(s)$.

The above claim completed the analysis of the algorithm for finding an integral distance- 2 transfer of a given tree instance and Lemma 6 follows.

\section{Better preprocessing for better algorithms}

In this section, we explore the possibility of a further improvement in the performance guarantee and integrality gap bounds via a better preprocessing. We demonstrate this by presenting a 6-approximation algorithm for the $\{0, L\}$-case of our problem. Formally, this is the special case of the capacitated $k$-center problem in which all the vertex capacities are either 0 or $L$, for some integer $L$. Instances with this property will be called $\{0, L\}$-instances.

It turns out that instances arising from the NP-hardness results, as well as the gap instances for the standard LP relaxation are all of this form, so this special case seems 
to capture the essential combinatorial difficulty of the capacitated problem. For these, we prove the following theorem:

Theorem 3 There is a polynomial-time algorithm achieving a 6-approximation for $\{0, L\}$-instances of the capacitated $k$-center problem.

General framework revisited. Let us recall the preprocessing done by Cygan et al. [10], explained in Sect. 2. The idea is to guess the optimum (call the guess $\tau$ ), and consider an unweighted graph $G_{\leq \tau}$ in which we place an edge between $u, v$ if $d(u, v) \leq \tau$. If we then solve the LP on such a graph, the integrality gap is unbounded, as the following example shows. We have two groups of 3 vertices, such that the distance within the groups is 1 , and distance between the groups is some large $C$. Suppose the capacity of each vertex is 2 , and $k=3$. Then the LP for the instance is feasible with $\tau=1$, while the OPT is $C$.

The trick to avoid this situation is to restrict to connected components of $G_{\leq \tau}$ defined above, then for each $i$, determine the smallest integer $k_{i}$ for which the LP is feasible in component $i$, and finally check if $\sum_{i} k_{i} \leq k$. (If not, the guess $\tau$ is too small). For connected graphs $G_{\leq \tau}$, our main theorem shows that the integrality gap is at most 9. Cygan et al. [10] gave a connected $\{0, L\}$-instance with integrality gap 7 , i.e., the $\operatorname{LP}_{k}\left(G_{\leq \tau}\right)$ is feasible with $\tau=1$, while OPT $\geq 7$.

So in a nutshell, the steps above can be seen as coming up with a graph (in this case $G_{\leq \tau}$ ) which has edges between $u, v$ only if it is feasible to assign $u$ to $v$ and vice versa, solving the LP on the connected components of this graph, and verifying that $\sum_{i} k_{i} \leq k$, as described. The aim of better preprocessing would then be to come up with a graph with even fewer edges, while still guaranteeing that the optimum assignment is preserved. Intuitively, this could produce more connected components, thus the $\sum_{i} k_{i} \leq k$ condition now becomes stronger.

For the $\{0, L\}$-case, we prove that an extremely simple additional preprocessing, namely removing edges between vertices $u$ and $v$ with $L(u)=L(v)=0$, provably lowers the integrality gap. Theorem 3 follows from Lemma 7:

Lemma 7 Suppose $G_{\leq \tau}^{*}$ is a connected component after the new preprocessing described above, and suppose $\operatorname{LP}_{k}\left(G_{\leq \tau}^{*}\right)$ is feasible, for some $k$. Then there is an algorithm to compute a set of $k$ vertices to open and an assignment of every vertex $u$ to an open center $v$ such that $d(u, v) \leq 6$, and the capacity constraints are satisfied.

The preprocessing leads to additional structure in the instance which we then use carefully in our rounding procedure. The proof is presented in Sect. 5.1. A natural open question is whether a similar approach can be applied to the general problem as well, improving our 9-approximation algorithm.

\subsection{Rounding procedure for the $\{0, L\}$-case}

In this section, we present the proof of Lemma 7 yielding the 6-approximation algorithm for the $\{0, L\}$-case. 
We call a vertex a 0 -node if its capacity is zero; an $L$-node otherwise. Let $V_{L}$ denote the set of $L$-nodes. $N^{L}[v]$ denotes $N[v] \cap V_{L}$. Let $G=(V, E)$ denote a connected component of $G_{\leq \tau}^{*}$ after the new preprocessing.

Recall that the 9-approximation algorithm rounds the opening variables of the LP solution "locally": it considers the tree of clusters in the bottom-up fashion, and for each subtree $T_{u}$, it opens $\left\lfloor y\left(T_{u}\right)\right\rfloor$ centers while deferring the decision of whether to open one additional center to the later subinstances. Our 6-relaxed decision procedure also operates as a bottom-up local rounding procedure, but in this case, our preprocessing ensures that a path from (the midpoint of) a child cluster to (the midpoint of) the parent does not contain consecutive 0 -nodes; this implies that $L$-nodes are very well "dispersed" throughout the graph, permitting local rounding to be performed at a finer granularity within closer proximity. In fact, even without such change in the granularity of rounding, a careful choice of $m_{v}$ alone with the original rounding algorithm is sufficient to give a 8-relaxed decision procedure.

Further improvements are facilitated by a better clustering. The clustering algorithm of Khuller and Sussmann [17] that is used by our 9-approximation algorithm finds cluster midpoints that are connected by length-three paths. This is in order to guarantee that $y\left(C_{v}\right) \geq 1$ for each cluster $C_{v}$, by ensuring $N[v] \subseteq C_{v}$. However, in a $\{0, L\}$ instance, $N^{L}[v] \subseteq C_{v}$ is sufficient to yield $y\left(N[v] \cap C_{v}\right) \geq 1$, and hence we can choose two vertices that are at distance 2 as cluster midpoints as long as all their common neighbors are 0 -nodes. This observation leads to an improved clustering where some parent and child can be closer.

Clustering algorithm. Our clustering algorithm runs in two phases. During the first phase, it identifies cluster midpoints one by one; each time a new cluster midpoint $v$ is identified, $N^{L}[v]$ is allotted to the new cluster $C_{v}$. The next cluster midpoint is always chosen at distance 2 from the set of already allotted vertices to ensure that $N^{L}[v]$ of each cluster are disjoint. Once the first phase is over, we may be left with some unallotted nodes. The second phase of the algorithm assigns every unalloted $L$-node to a nearby cluster.

Algorithm 1 shows our clustering algorithm. Let $\alpha(u)$ for each allotted node $u$ denote the midpoint of the cluster to which $u$ is allotted: $u \in C_{\alpha(u)}$. During the first phase, $V_{\text {allotted }}$ maintains the set of vertices that has been already allotted to a cluster by the algorithm; $\operatorname{dist}(v)$ denotes the shortest distance from $V_{\text {allotted }}$ to $v$ : $\operatorname{dist}(v):=\min _{u \in V_{\text {allotted }}} d_{G}(u, v)$. In addition to identifying cluster midpoints, our algorithm also chooses $p(v) \in C_{v}$ for each cluster $C_{v}$, on which the opening of one is to be aggregated later. Also, for each non-root cluster $C_{v}$, the algorithm finds a vertex in the parent cluster through which $v$ is connected to the parent cluster and call it $\pi_{1}(v)$.

After the first phase, $V_{\text {allotted }}$ retains its final value, i.e., the set of all vertices allotted during the first phase. $V_{\text {allotted }}$ does not change any more, and so neither does dist. The second phase assigns each unallotted $L$-node $v$ to a nearby cluster; it also annotates $v$ with $\pi_{2}(v)$, where $\pi_{2}(v)$ denotes the vertex through which $v$ is connected to $\alpha(v)$. Figure 3 shows an example of an execution of Algorithm 1. 


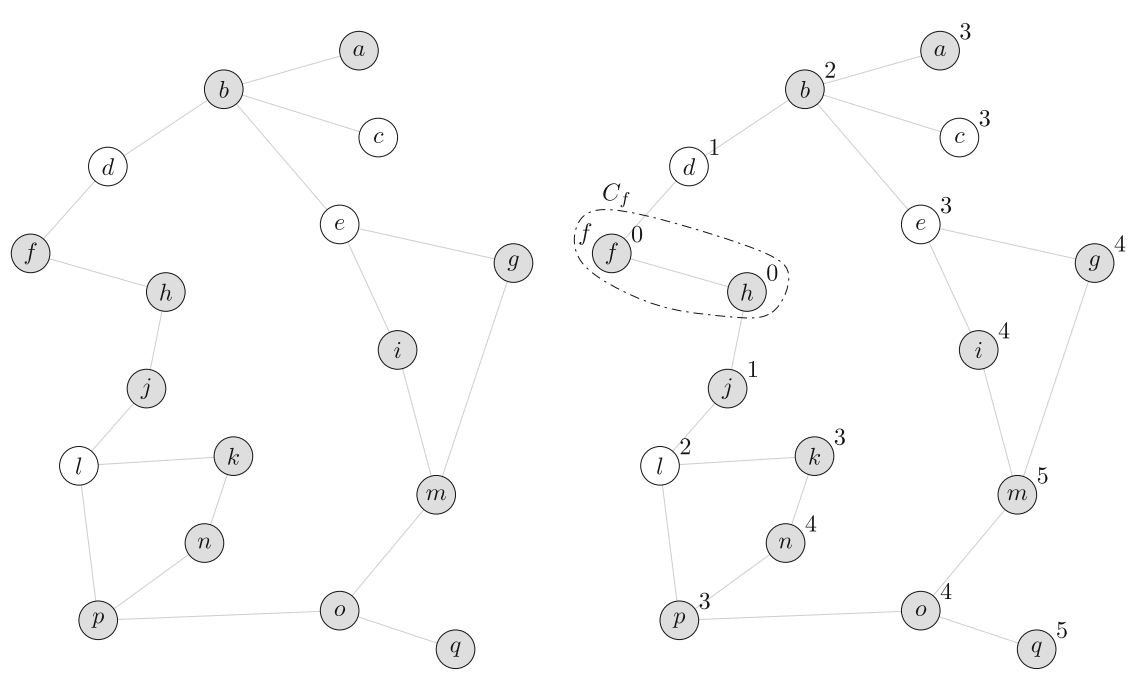

(a)

(b)

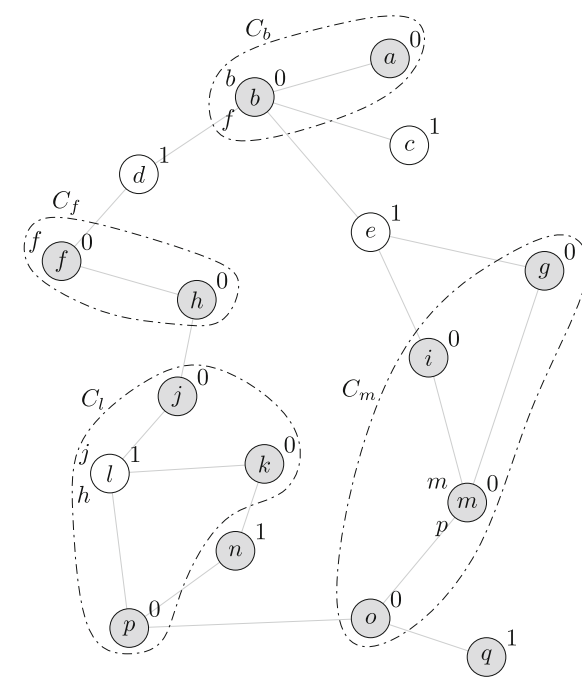

(c)

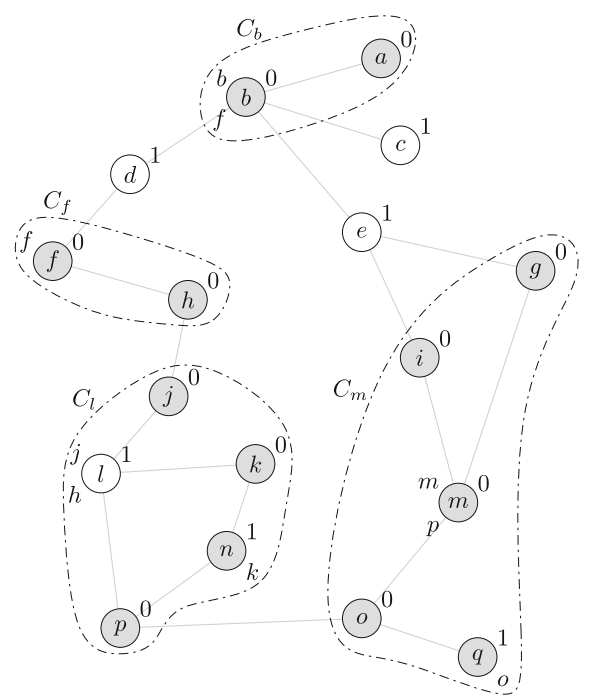

(d)

$$
{ }_{\pi_{1}(v)}^{p(v)}(v)_{\pi_{2}(v)}^{\operatorname{dist}(v)}
$$

(e)

Fig. 3 Example of an execution of Algorithm 1. L-nodes are represented as shaded circles, and 0-nodes are empty circles. For each vertex $v$, $\operatorname{dist}(v), p(v), \pi_{1}(v)$, and $\pi_{2}(v)$ is respectively shown on the upper right, upper left, lower left, and lower right corner of the vertex, if defined. Clusters are shown as closed curves; $\alpha(v)$ is not shown since it simply is the shorthand for " $u$ such that $v \in C_{u}$ ". a Given graph $G$. b At the end of Step 4. $\mathbf{c}$ At the end of the first phase. $\mathbf{d}$ At the end of the second phase. e Legend 


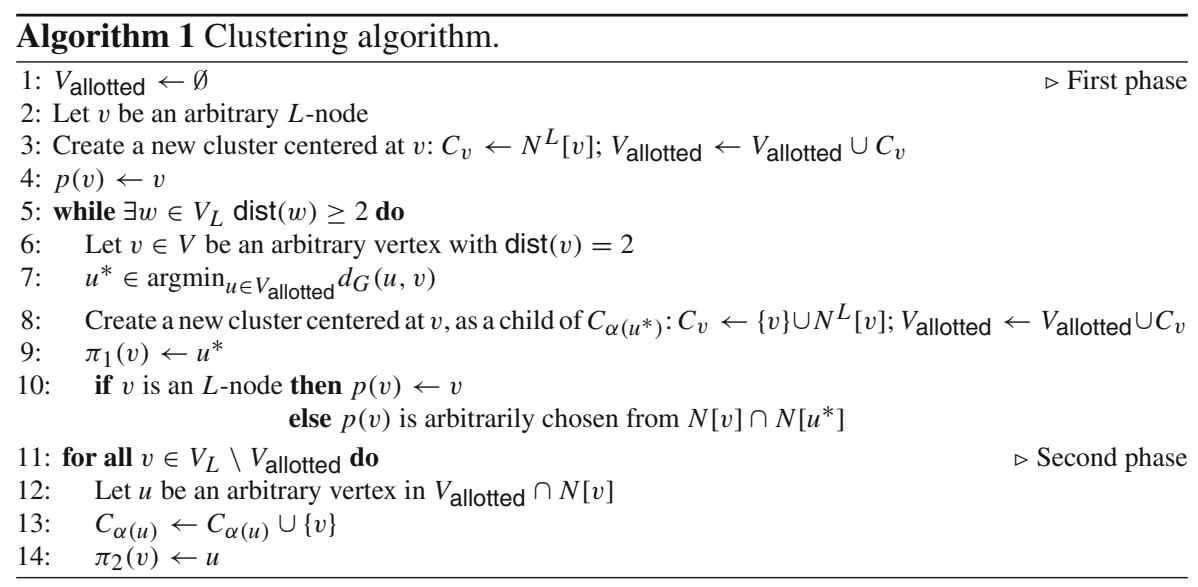

Lemma 8 Algorithm 1 is well-defined, and its output satisfies the following:

(i) $N^{L}[v] \subseteq C_{v}$ for every $C_{v}$, and $C_{v}$ 's are disjoint;

(ii) every L-node is allotted to some cluster, and a 0-node is allotted only when it becomes a cluster midpoint;

(iii) $p(v) \in N^{L}[v]$ for every $C_{v}$;

(iv) $\pi_{1}(v)$, when defined, is in $N^{L}[x]$ for some $C_{x} ; \pi_{2}(v)$, when defined, is in $N^{L}[y]$ for some $C_{y}$;

(v) $C_{v}= \begin{cases}N^{L}[v] \cup\left\{u \mid \pi_{2}(u) \in N^{L}[v]\right\}, & \text { if } v \text { is an } L \text {-node; } \\ \{v\} \cup N^{L}[v] \cup\left\{u \mid \pi_{2}(u) \in N^{L}[v]\right\}, & \text { if } v \text { is a } 0 \text {-node. }\end{cases}$

Proof Since $\operatorname{LP}_{k}(G)$ is feasible, $V_{L} \neq \varnothing$ and $v$ can be chosen at Step 2. At Step 6, as there exists $w \in V_{L}$ with $\operatorname{dist}(w) \geq 2$, there exists a vertex $v$ with $\operatorname{dist}(v)=2$, for example the one that appears on a path of length $\operatorname{dist}(w)$ from $V_{\text {allotted }}$ to $w$. Note that $v \in V$ may be a 0 -node or an $L$-node. At Step $10, d_{G}\left(u^{*}, v\right)=2$ from the choice of $u^{*}$ and hence $N[v] \cap N\left[u^{*}\right]$ is nonempty. When the while loop terminates, $\operatorname{dist}(w) \leq 1$ for every $w \in V_{L}$; thus, $v$ at Step 12 satisfies $\operatorname{dist}(v)=1$ and therefore $u$ can be chosen. The algorithm is well-defined.

Each time a new cluster $C_{v}$ is created, $N^{L}[v]$ is added to $C_{v}: N^{L}[v] \subseteq C_{v}$. The only two cases in which we create a new cluster $C_{v}$ is when it is the first cluster created, and when $\operatorname{dist}(v)=2$. In the latter case, $\operatorname{since} \operatorname{dist}(v)=2, N^{L}[v] \cap V_{\text {allotted }}=\emptyset$ and therefore $\{v\} \cup N^{L}[v]$ is disjoint from $V_{\text {allotted }}$, the set of currently allotted vertices. Thus, at the end of the first phase, $C_{v}$ 's are disjoint. No new clusters are created during the second phase and only the $L$-nodes that has not been allotted are added to exactly one of the existing clusters. Hence, Property (i) holds.

Property (ii) is easily verified, since Steps 11-14 ensure that every $L$-node is allotted, and the only case a 0 -node is allotted is at Step 8, where the cluster midpoint $v$ is allotted.

At Step 10, if $v$ is a 0-node, $N[v] \backslash\{v\} \subseteq V_{L}$ since every edge is incident to at least one $L$-node; Property (iii) follows from this observation. 
During the first phase, vertices are allotted only when it is a cluster midpoint or in $N^{L}[v]$ for some cluster midpoint $v$. Thus, $u^{*} \in V_{\text {allotted }}$ chosen at Step 7 is either a cluster midpoint or in $N^{L}[v]$ for some $C_{v}$. Suppose $u^{*}$ is a cluster midpoint. If $u^{*}$ is an $L$-node, then $u^{*} \in N^{L}\left[u^{*}\right]$; suppose $u^{*}$ is a 0-node. As $d_{G}\left(u^{*}, v\right)=2$ from the choice of $u^{*}$, there exists a vertex $z$ that is in both $N\left[u^{*}\right]$ and $N[v] . z$ is an $L$-node since $u^{*}$ is a 0 -node. Thus $z$ is in $N^{L}\left[u^{*}\right]$ and has to be in $V_{\text {allotted }}$, contradicting $\operatorname{dist}(v)=2$. Hence, in any case, $\pi_{1}(v) \in N^{L}[x]$ for some $C_{x}$, verifying the first half of Property (iv).

At Step 12, $u \in V_{\text {allotted }}$ and hence either $u$ is a cluster midpoint or $u \in N^{L}[y]$ for some $C_{y}$. If $u$ is a cluster midpoint, $v \in N^{L}[u]$, contradicting $v \notin V_{\text {allotted. This }}$ completes the verification of Property (iv).

At the end of the first phase, for every $C_{v}, C_{v}=\{v\} \cup N^{L}[v]$ from construction and it can be only augmented in the rest of the algorithm. When $v$ is added to a cluster at Step 13, it is added to $C_{\alpha\left(\pi_{2}(v)\right)}$ and hence

$$
C_{v}= \begin{cases}N^{L}[v] \cup\left\{u \mid \pi_{2}(u) \in\{v\} \cup N^{L}[v]\right\}, & \text { if } v \text { is a } L \text {-node; } \\ \{v\} \cup N^{L}[v] \cup\left\{u \mid \pi_{2}(u) \in\{v\} \cup N^{L}[v]\right\}, & \text { if } v \text { is a } 0 \text {-node. }\end{cases}
$$

Now Property (v) follows from Property (iv).

Observation 4 For every non-root cluster $C_{v}$, the distance between $\pi_{1}(v)$ and $p(v)$ is

$$
\begin{cases}1, & \text { if } v \text { is a } 0 \text {-node; } \\ 2, & \text { otherwise. }\end{cases}
$$

Proof Note that $\pi_{1}(v)$ and $v$ are at distance 2 as can be seen from Step 7 of Algorithm 1; thus, if $v$ is an $L$-node, $p(v)=v$ and the distance between $\pi_{1}(v)$ and $p(v)$ is two. If $v$ is a 0 -node, $p(v)$ is chosen from $N\left[\pi_{1}(v)\right]$ at Step 10 of Algorithm 1.

Rounding opening variables. Our algorithm will gradually round the opening variables $y$, starting from the original LP solution, until they become integral. This process will be described in terms of opening movements, where each movement specifies how much opening is moved from which $L$-node to which $L$-node. Since the $L$-nodes have the same capacities, if we show that a set of opening movements makes the opening variables integral while no opening is moved by the net distance of more than $r$, this implies that the resulting set of opening variables is an integral distance- $r$ transfer.

Our rounding procedure begins with changing $y_{p(v)}$ of every cluster $C_{v}$ to one: for each cluster $C_{v}$, we increase $y_{p(v)}$ until it reaches one, while simultaneously decreasing the opening variable of a vertex in $N^{L}[v]$ by the same amount. This initial aggregation can be interpreted as opening movements, and keeps the budget constraint $\sum_{v \in V} y_{v}=$ $k$ satisfied. 
Observation 5 For each cluster $C_{v}$, the initial aggregation can be implemented by a set of opening movements within the distance of

$$
\begin{cases}1, & \text { if } p(v)=v \\ 2, & \text { otherwise. }\end{cases}
$$

Proof $N^{L}[v]$ 's are disjoint from Lemma 8, and $y\left(N^{L}[v]\right) \geq 1$ from the LP constraints; hence, $y_{p(v)}$ can be made 1 via movements from $N^{L}[v]$. Note that $p(v) \in N^{L}[v]$ in any case.

After the initial aggregation, the procedure considers each cluster $C_{v}$ in the bottomup order and make the opening variables of every vertex in $C_{v} \backslash\{p(v)\}$ integral, using movements of distance 5 or smaller; $p(v)$ is propagated to the parent cluster, to be taken into account when that cluster is rounded. Precisely, the rounding procedure for $C_{v}$ rounds the opening variables of $I_{v}:=V_{L} \cap\left(C_{v} \backslash\{p(v)\} \cup\left\{p(u) \mid \pi_{1}(u) \in C_{v}\right\}\right)$, i.e., the set of $L$-nodes that is either propagated from a child cluster or originally in $C_{v}$, except the vertex to be propagated from $C_{v}$.

Algorithm 2 shows the procedure. First it recursively processes the children clusters, and then constructs a family of vertex sets $\left\{X_{u}\right\}_{u \in N^{L}[v]}$ indexed by $N^{L}[v]$. For $u \neq$ $p(v), X_{u}$ consists of $u$ itself, vertices propagated from the children clusters that are connected through $u$, and the vertices in $C_{v}$ that are connected to $v$ through $u: X_{u}:=$ $\{u\} \cup\left\{p(w) \mid \pi_{1}(w)=u\right\} \cup\left\{w \mid \pi_{2}(w)=u\right\} . X_{p(v)}$ is similarly defined, except that it does not contain $p(v)$. Now for every $u \in N^{L}[v]$, we locally round $X_{u}$ : we choose a set $W_{u}$ of the vertices to be opened, and move the openings of the other vertices to the vertices in $W_{u}$. Note that LocaLRound $\left(V_{\text {toOpen }}, V_{\text {moveFrom1 }}, V_{\text {moveFrom2 }}\right)$ is a procedure that increases the opening variables of the vertices in $V_{\text {toOpen }}$ to one, while decreasing the opening variables of $V_{\text {moveFrom1 }}$ (and $V_{\text {moveFrom2 }}$ if $V_{\text {moveFrom1 }}$ is used up) to match the increase. $W_{u}$ is chosen as a subset of $X_{u}$, but we avoid choosing $u \in N^{L}[v]$ whenever possible. After these local roundings, each $X_{u}$ may still have some non-integral opening variables remaining; we choose a set $F \subseteq N^{L}[v] \backslash\{p(v)\}$ to accomodate these openings. Finally, if there still remains some fraction, we choose one last center denoted by $w^{*}$, and open it using the opening movements from the vertices that are still fractionally open (denoted by $\bar{I}_{\text {Step12 }}$ ) and $p(v)$. Note that $y_{p(v)}$, therefore, may become less than one at the termination of $\operatorname{RounD}(v)$.

Lemma 9 Suppose that $y_{p(v)}=1$ before Step 3 of $\operatorname{Round}(v)$. Then Steps 3-15 of $\operatorname{Round}(v)$ make the opening variables of $I_{v}$ integral, and this can be implemented by a set of opening movements within $I_{v} \cup\{p(v)\}$, with no incoming movements to $p(v)$. The maximum distance of these movements is five taking the initial aggregation into account.

Proof Note that, from Properties (iii), (iv), and (v) of Lemma 8, $\left\{X_{u}\right\}_{u \in N^{L}[v]}$ forms a partition of $I_{v}$. Thus it suffices to verify that the opening variables of each $X_{u}$ becomes integral. Also note that $X_{u} \subseteq V_{L}$.

At Step 6 of the algorithm, we have $y\left(X_{u}\right) \leq\left|X_{u}\right|$ from $y \leq \mathbf{1}$; hence $W_{u}$ can be successfully chosen. After Step 7, $X_{u}$ may still have some non-integral opening 


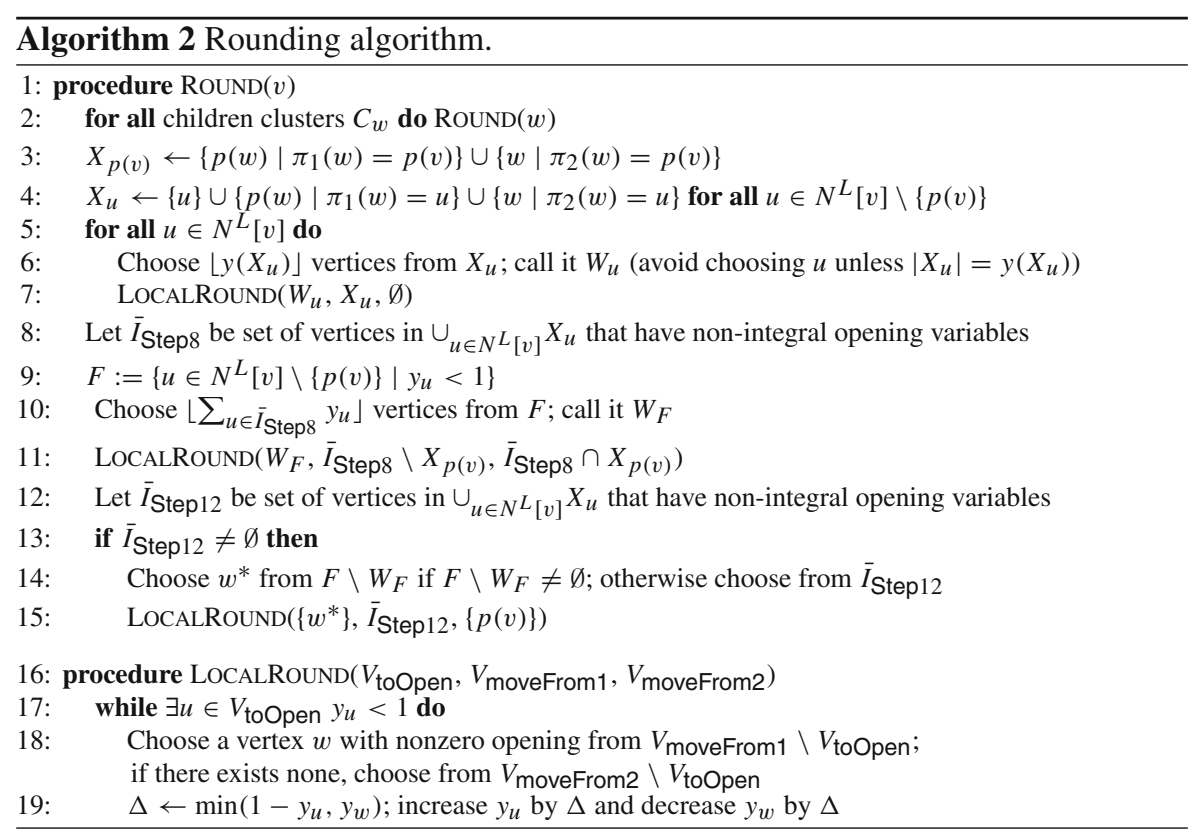

variables, but their total opening is given by $r_{u}:=y\left(X_{u}\right)-\left\lfloor y\left(X_{u}\right)\right\rfloor<1$. Moreover, when $r_{u}>0$, we have $u \notin W_{u}$ and therefore $y_{u}<1$. Thus, $\sum_{u \in \bar{I}_{\text {Step } 8}} y_{u}=r_{p(v)}+$ $\sum_{u \in N^{L}[v] \backslash\{p(v)\}} r_{u}<1+|F|$ at Step 10 and therefore $W_{F}$ can be chosen as well. Note that $\left(\bar{I}_{\text {Step8 }} \backslash X_{p(v)}\right) \cup\left(\bar{I}_{\text {Step8 }} \cap X_{p(v)}\right)=\bar{I}_{\text {Step8 }}$ and hence Step 18 of LOCALRoUND called from Step 11 will always succeed. After Step 11, the total non-integral opening variables in $I_{v}$ will become strictly smaller than one. If $\bar{I}_{\text {Step } 12}=\emptyset$, we are done. Otherwise, $w^{*}$ can be successfully chosen since $\bar{I}_{\text {Step } 12} \neq \emptyset$, and Step 15 will make the opening variables of $I_{v}$ completely integral, while making $y_{p(v)}$ smaller than one. Note that $y_{p(v)}=1$ before Step 15 .

Now it remains to verify that this rounding can be realized in terms of opening movements within the distance of five. When $x \in X_{u}$, one of the following holds: (i) $x=u$, (ii) $x=p(w)$ and $\pi_{1}(w)=u$, or (iii) $\pi_{2}(x)=u$. In Case (ii), $d_{G}(x, u) \leq 2$ from Observation 4. In Case (iii), $d_{G}(x, u)=1$ as can be seen from Step 12 of Algorithm 1. Thus, for any $x \in X_{u}, x$ is within the distance of 2 from $u$. Note that the opening at $x \in X_{u}$ has been moved from $N^{L}[w]$ if $x=p(w)$; otherwise, it originates from $x$ itself. From Observations 4 and 5, the opening at $x$, in Case (ii), originates from vertices within the distance of three from $u$; in the other cases, it is from $x$ itself and therefore within the distance of one. Thus, any movements resulting from Step 7 of Algorithm 2 moves opening that originally comes from vertices within the distance of three from $u$ to a vertex within the distance of two from $u$; the maximum distance of these movements therefore is five.

Since the opening at $x \in X_{u}$ originates from vertices within the distance of three from $u$, it is within the distance of four from $v$. On the other hand, every vertex in $F$ 
is within the distance of one from $v$; therefore, the maximum distance of movements resulting from Step 11 also is five.

Suppose $w^{*}$ is chosen from $F \backslash W_{F}$ at Step 14. Then $w^{*}$ is within the distance of one from $v$; as observed earlier, the opening at $x \in \bar{I}_{\text {Step } 12}$ originates from vertices within the distance of four from $v \cdot p(v)$ is within the distance of one from $v$, and its opening originates from vertices within the distance of two from $p(v)$ (see Observation 5); hence, the opening at $p(v)$ originates from vertices within the distance of three from $v$. Thus, in this case, any movements resulting from Step 15 moves opening that originates from vertices within the distance of four from $v$ to a vertex within the distance of one from $v$; the maximum distance of these movements therefore is five.

Suppose $F \backslash W_{F}=\emptyset$. In this case, $F=W_{F}$ and thus $\sum_{u \in \bar{I}_{\text {Step }>} \backslash X_{p(v)}} y_{u}<|F|=$ $\left|W_{F}\right|$. Hence, $\bar{I}_{\text {Step } 8} \backslash X_{p(v)}$ is used up during LoCALRound called from Step 11 (note that $\left.\left(W_{F} \cap \bar{I}_{\text {Step } 8}\right) \subseteq\left(\bar{I}_{\text {Step } 8} \backslash X_{p(v)}\right)\right)$. Therefore, we have $\bar{I}_{\text {Step12 }} \subseteq X_{p(v)}$. As observed earlier, $w^{*} \in X_{p(v)}$ is within the distance of two from $p(v)$; the opening at $x \in X_{p(v)}$ is from vertices within the distance of three from $p(v)$. The opening at $p(v)$ is from vertices within the distance of two from $p(v)$, as was seen in Observation 5. Thus, the maximum distance of movements resulting from Step 15 is five in this case as well.

Proof (of Lemma 7) Let $C_{r}$ be the root cluster, and we execute Round $(r)$ on the LP solution.

From Lemma 9, Round $(r)$ outputs a set of opening variables that can be realized by a set of opening movements of distance five or smaller: note that $y_{p(v)}=1$ before Step 3 of each execution of $\operatorname{Round}(v)$, since we process the clusters in the bottomup order. As every vertex in $V_{L} \backslash\{p(r)\}$ is in $I_{v}$ for some cluster $C_{v}$, their opening variables are made integral. Since $\sum_{v \in V} y_{v}=k, y_{p(r)}$ is also made integral, and the opening movements to $p(r)$ during the initial aggregation were within the distance of one. Thus the output set of open vertices is an integral distance- 5 transfer.

Now Lemma 2 completes the proof.

\section{Extensions to other problems}

In this section, we present how our techniques readily apply to two other problems.

\subsection{Capacitated $k$-supplier}

Firstly, we present an 11-approximation algorithm for the capacitated $k$-supplier problem. This problem is a generalization of the capacitated $k$-center problem in which some vertices are designated clients and some facilities. We can only open $k$ of the facilities, and the aim is to serve the clients (facilities do not have to be served).

Let us denote by $\mathscr{C}$ and $\mathscr{F}$ the set of clients and facilities respectively. For this version, we prove the following.

Theorem 6 There exists a polynomial time 11-approximation algorithm for the capacitated $k$-supplier problem. 
The algorithm proceeds along the lines of our main result. We first guess the optimum $\tau$, and restrict to the bipartite graph $G$ on vertex sets $\mathscr{C}, \mathscr{F}$, with an edge between $u \in \mathscr{C}$ and $v \in \mathscr{F}$ iff $d(u, v) \leq \tau$. We then divide this into connected components and work with them separately, as before. Thus in what follows, let us assume that $G$ as defined above is connected, and $\operatorname{LP}_{k}(G)$ is feasible. Note that this is a slightly different LP, where $y$-variables exist only for facilities, and the constraints $\sum_{u:(u, v) \in E} x_{u v}=1$ exist only for the clients.

The main difference in this variant is in the clustering step. We modify the clustering of Khuller and Sussmann [17] as follows. Start with an arbitrary client $u \in \mathscr{C}$ as the first cluster midpoint, and include all of $N[u]$ in the cluster $C_{u}$. Now as long as possible, do the following: pick a client $u \in \mathscr{C}$ which is at a distance $>2$ from the midpoints of all the clusters so far, but is distance precisely 4 from some cluster midpoint; include all of $N[u]$ into the new cluster $C_{u}$ (there will not be an overlap with other clusters because of the distance condition).

When the procedure ends, we will be left with a bunch of clients at distance 2 from some cluster midpoints, and some facilities at distance 3 from some cluster midpoints (and nothing else, by connectivity properties). We move them to the closest cluster (breaking ties arbitrarily). Now the procedure satisfies the following conditions:

1. Each cluster has its $y$-values adding up to $\geq 1$ (indeed, the neighborhood of the cluster midpoint has total $y$-value $\geq 1$, as is required in the tree reduction).

2. The graph of clusters, in which we place an edge if the midpoints are at distance precisely 4 , is connected.

These properties ensure that we can perform precisely the same reduction to tree instances, however we have a variant of Lemma 3: an $r$-transfer to the tree instance now implies a $(4 r+3)$ approximation algorithm for the client/facility problem. This is because adjacent cluster midpoints are at a distance 4 , and hence the distance in $G$ between two vertices $a_{u}$ and $a_{v}$ (as in the reduction) which have distance $r$ in the tree instance, is now $4 r$. The rest of the proof carries over verbatim, and we obtain a reduction to tree instances with the above guarantee.

This proves Theorem 6, because for tree instances, we can use our algorithm which gives $r=2$.

\subsection{Budgeted version with uniform capacities}

The budgeted center problem is a weighted generalization of the $k$-center problem: in the $k$-center problem, opening a center incurs the uniform cost of one and there is a budget of $k$ on the total opening cost; on the other hand, in the budgeted center problem, the opening costs are given by $C: V \rightarrow \mathbb{R}_{+}$that is a part of the input along with the total budget $B \in \mathbb{R}_{+}$. When the capacities are uniform, Khuller and Sussmann [17], using the technique of Bar-Ilan et al. [4], gives a 13-approximation algorithm. In this subsection, we present a 9-approximation algorithm for the budgeted center problem with uniform capacities. We note that it is easy to extend this result to the $\{0, L\}$-case as well.

Let $L_{0} \in \mathbb{N}$ be the uniform capacity. Following is the key lemma of our analysis: 
Lemma 10 Suppose there exists a polynomial-time algorithm that finds an integral distance-r transfer of a tree instance. Then there exists an algorithm that, given a connected graph $G=(V, E)$, the constant capacity function $L: V \rightarrow\left\{L_{0}\right\}$, and $k \in \mathbb{N}$ for which $\operatorname{LP}_{k}(G)$ has a feasible solution $(x, y)$, in addition to the opening costs $C: V \rightarrow \mathbb{R}_{+}$, finds an integral distance- $(3 r+2)$ transfer $y^{\prime}$ of $(G, L, y)$ satisfying $\sum_{v \in V} C(v) y_{v}^{\prime} \leq \sum_{v \in V} C(v) y_{v}$.

Our 9-approximation algorithm follows from Lemma 10.

Theorem 7 There exists a 9-approximation algorithm for the budgeted center problem with uniform capacities.

Proof Let OPT denote the optimal solution value. As in Lemma 1, our algorithm makes a guess $\tau$ at the optimal solution value and tries to decide if $\tau<$ OPT. In this problem again, we consider the graph $G_{\leq \tau}$ representing the admissible assignments. Consider the connected components of $G_{\leq \tau}$; for each component $G_{i}$, we will compute a lower bound $B_{i}$ on the minimum budget necessary to have a feasible solution to the subproblem induced by $G_{i}$. Observe that, if $\tau \geq$ OPT, an optimal solution assigns every vertex to a center that is in the same connected component. Thus, if $\sum_{i} B_{i}>B$, we can certify that $\tau<$ OPT. $B_{i}$ is determined by solving $\operatorname{LP}_{k_{i}}\left(G_{i}\right)$, but with an objective of minimizing the opening cost $\sum_{v \in G_{i}} C(v) y_{v}$ rather than as a feasibility LP with no objective function; $k_{i}$ is chosen by trying all integers from 1 to $\left|V\left(G_{i}\right)\right|$ and selecting the one that gives the smallest opening cost. If we failed to certify $\tau<$ OPT, this means $\sum_{i} B_{i} \leq B$. Now for each $G_{i}$, Lemmas 2 and 10 let us find a set of vertices to open for which there exists an assignment of every vertex to an open center that is within the distance of $3 r+3$, and the total opening cost of this set is no greater than $B_{i}$. The union of these sets is the desired solution from the triangle inequality. Recall that $r$ can be taken as two, from Lemma 6 .

Proof (of Lemma 10) We invoke our rounding procedure for the $k$-center problem, but with the "fake" capacity function $\hat{L}$ defined as $\hat{L}(v):=\bar{C}_{\max }-C(v)$, where $\bar{C}_{\max }:=$ $1+\max _{v \in V} C(v)$. The output vector $y^{\prime}$ is an integral distance- $(3 r+2)$ transfer of $(G, \hat{L}, y)$ from Lemma 4 . Since $y^{\prime}$ is a distance- $(3 r+2)$ transfer, we have $\sum_{v \in V} y_{v}=$ $\sum_{v \in V} y_{v}^{\prime}=k$, and by taking $U=V$ in Condition (1b) of Definition 1, we also have $\sum_{v \in V} \hat{L}(v) y_{v}^{\prime} \geq \sum_{v \in V} \hat{L}(v) y_{v}$. Since $\sum_{v \in V} \hat{L}(v) y_{v}^{\prime}=\bar{C}_{\max } \cdot k-\sum_{v \in V} C(v) y_{v}^{\prime}$ and $\sum_{v \in V} \hat{L}(v) y_{v}=\bar{C}_{\max } \cdot k-\sum_{v \in V} C(v) y_{v}$, this implies that $\sum_{v \in V} C(v) y_{v}^{\prime} \leq$ $\sum_{v \in V} C(v) y_{v}$.

On the other hand, one can see that the decisions made by our rounding procedure purely depend on the relative ordering of capacities, rather than their actual values. Hence, the complete "execution history" of the rounding procedure with $\hat{L}$ could also be interpreted as a valid execution history with the true capacity function $L$ as well: if the procedure is executed with $L$, every comparison of capacities will always be a tie since $L$ is a constant function, and we can break them so that it will be consistent with the ordering of $\hat{L}$. Therefore, it is possible that our rounding algorithm outputs $y^{\prime}$ when it is run with $L$, and from Lemma $4, y^{\prime}$ is an integral distance- $(3 r+2)$ transfer of $(G, L, y)$. 


\section{Conclusions and future directions}

As was demonstrated in Sects. 6.1, 6.2, our techniques can be extended to obtain approximation algorithms for different problems. We see this as further evidence that the simplicity of our approach helps in designing better algorithms also for other location problems.

As our 9-approximation algorithm comes close to settling the integrality gap, it is natural to ask if our techniques can be used to obtain a tight result. Recall that our framework consists of first reducing the general problem to tree instances and then solving such instances. Since our algorithm for tree instances is the best possible, any potential improvement must come from the reduction, and we raise this as an open problem.

Finally, as the $\{0, L\}$-case suggests, our preliminary results on additional preprocessing indicate that further investigation is necessary to understand if these techniques can help bring down the integrality gap to the tight factor of 3. More generally, we believe that it is important not only for capacitated $k$-center but also for other problems, such as facility location and $k$-median, to understand the power of lift-andproject methods (applied to potentially different formulations). For example, do they automatically capture these preprocessing steps and lead to stronger formulations?

Acknowledgments The authors thank the anonymous reviewers of this paper and of its preliminary version [1] for their helpful comments.

\section{References}

1. An, H.C., Bhaskara, A., Chekuri, C., Gupta, S., Madan, V., Svensson, O.: Centrality of trees for capacitated $k$-center. In: IPCO, pp. 52-63 (2014)

2. Arya, V., Garg, N., Khandekar, R., Meyerson, A., Munagala, K., Pandit, V.: Local search heuristics for $k$-median and facility location problems. SIAM J. Comput. 33(3), 544-562 (2004)

3. Bansal, M., Garg, N., Gupta, N.: A 5-approximation for capacitated facility location. In: ESA, pp. 133-144 (2012)

4. Bar-Ilan, J., Kortsarz, G., Peleg, D.: How to allocate network centers. J. Algorithm. 15(3), 385-415 (1993)

5. Byrka, J.: An optimal bifactor approximation algorithm for the metric uncapacitated facility location problem. In: Approx-Random, pp. 29-43 (2007)

6. Charikar, M., Guha, S.: Improved combinatorial algorithms for facility location problems. SIAM J. Comput. 34(4), 803-824 (2005)

7. Charikar, M., Guha, S., Tardos, É., Shmoys, D.B.: A constant-factor approximation algorithm for the k-median problem. J. Comput. Syst. Sci. 65(1), 129-149 (2002)

8. Chudak, F.A., Williamson, D.P.: Improved approximation algorithms for capacitated facility location problems. Math. Program. 102(2), 207-222 (2005)

9. Chuzhoy, J., Rabani, Y.: Approximating $k$-median with non-uniform capacities. In: SODA, pp. 952-958 (2005)

10. Cygan, M., Hajiaghayi, M., Khuller, S.: LP rounding for k-centers with non-uniform hard capacities. In: FOCS, pp. 273-282 (2012)

11. Gonzalez, T.F.: Clustering to minimize the maximum intercluster distance. Theor. Comput. Sci. 38, 293-306 (1985)

12. Guha, S., Khuller, S.: Greedy strikes back: improved facility location algorithms. J. Algorithm. 31(1), 228-248 (1999)

13. Hall, P.: On representatives of subsets. J. Lond. Math. Soc. 10, 26-30 (1935) 
14. Hochbaum, D.S., Shmoys, D.B.: A best possible heuristic for the $k$-center problem. Math. Oper. Res. 10, 180-184 (1985)

15. Jain, K., Mahdian, M., Saberi, A.: A new greedy approach for facility location problems. In: STOC, pp. 731-740 (2002)

16. Jain, K., Vazirani, V.V.: Approximation algorithms for metric facility location and $k$-median problems using the primal-dual schema and lagrangian relaxation. J. ACM 48(2), 274-296 (2001)

17. Khuller, S., Sussmann, Y.J.: The capacitated $k$-center problem. SIAM J. Discret. Math. 13(3), 403-418 (2000)

18. Korupolu, M.R., Plaxton, C.G., Rajaraman, R.: Analysis of a local search heuristic for facility location problems. J. Algorithm. 37(1), 146-188 (2000)

19. Levi, R., Shmoys, D.B., Swamy, C.: LP-based approximation algorithms for capacitated facility location. In: IPCO, pp. 206-218 (2004)

20. Li, S.: A 1.488 approximation algorithm for the uncapacitated facility location problem. In: ICALP (2), pp. 77-88 (2011)

21. Li, S., Svensson, O.: Approximating $k$-median problem via pseudo-approximation. In: STOC, pp. 901-910 (2013)

22. Pál, M., Tardos, É., Wexler, T.: Facility location with nonuniform hard capacities. In: FOCS, pp. 329-338 (2001)

23. Shmoys, D.B., Tardos, É., Aardal, K.: Approximation algorithms for facility location problems (extended abstract). In: STOC, pp. 265-274 (1997)

24. Williamson, D.P., Shmoys, D.B.: The Design of Approximation Algorithms. Cambridge University Press, New York (2011)

25. Zhang, J., Chen, B., Ye, Y.: A multiexchange local search algorithm for the capacitated facility location problem. Math. Oper. Res. 30(2), 389-403 (2005) 